\title{
MicroRNAs provide the first evidence of genetic link between diapause and aging in vertebrates
}

\author{
Luca Dolfi ${ }^{1^{*}}$ Mario Baumgart ${ }^{2} *$, Marco Groth ${ }^{2}$, Roberto Ripa ${ }^{1}$, Matthias Platzer ${ }^{2}$ and Alessandro \\ Cellerino $^{1,2}$
}

1 Scuola Normale Superiore, Pisa, Italy; 2 Fritz Lipmann Institute for Age Research, Leibniz Institute, Jena

All correspondence should be sent to. Alessandro Cellerino, Scuola Normale Superiore Pisa. c/o Istituto di Biofisica, CNR, via Moruzzi 1, 56124 Pisa Italy, tel: 00390503152756; email a.cellerino@sns.it

Running title: miRNAs and vertebrate diapause

*equal contribution

\section{Summary}

Diapause and aging are controlled by overlapping genetic mechanisms in C.elegans and these include microRNAs (miRNAs). Here, we investigated miRNA regulation in embryos of annual killifish that naturally undergo diapause to overcome desiccation of their habitats. We compared miRNA expression in diapausing and non-diapausing embryos in three independent lineages of killifish. We identified 13 miRNAs with similar regulation in all three lineages. One of these is miR-430, which is known as key regulator of early embryonic development in fish. We further tested whether this regulation overlaps with the aging-dependent regulation of miRNAs in one annual species: Nothobranchius furzeri. We found that miR-101a and miR-18a are regulated in the same direction during diapause and aging. These results provide the first evidence that overlapping genetic networks control diapause and aging in vertebrates and suggest that diapause mimics aging to some extent..

Diapause is a suspension of larval development to overcome adverse conditions. An overlap in the genetic mechanisms controlling diapause (dauer) and longevity was discovered in C. elegans and led 
to the identification of insulin/IGF pathways as a conserved regulator of aging (Kenyon 2011). MicroRNAs (miRNAs) are part of the genetic network that regulates longevity and diapause (de Lencastre et al. 2010; Pincus et al. 2011; Zhang et al. 2011). These results prompted us to investigate a possible overlap in microRNA regulation during aging and diapause in vertebrates.

Diapause is a survival strategy in annual fish that are adapted the alternation of wet- and dry-season in Africa and South America (Wourms 1972) and is present in three independent evolutionary lineages of killifishes as the result of multiple events of loss and re-gain of an ancestral annual life history (Murphy \& Collier 1997; Hrbek \& Larson 1999). In each of these clades, annual species have as nearest neighbor a non-annual species (Fig. 1A) and this taxon offers a unique paradigm to test parallel evolution of life-history traits.

Aim of the present study was to characterize the miRNA signature of diapause in annual fish and compare it with the signature we previously detected during aging of the annual species Nothobranchius furzeri (Baumgart et al. 2012).

We used miRNA-Seq (Morin et al. 2008) to identify and quantify expression of miRNAs during diapause. We analyzed diapausing embryos and embryos in the corresponding developmental stage (mid-somite) for five annual species and three non-annual species from all three lineages (Fig.1A). We used hierarchial clustering (Fig. 1C) and multi dimensional scaling (MDS, Fig. 1B) to visualize global aspects of miRNA regulation. Results showed that samples cluster according to their physiological status (diapausing or not) and not to their phylogenetic relationships. Similarly, diapausing and non-diapausing samples occupy non-overlapping regions of the MDS space.

We identified miRNAs differentially expressed between closely related annual and non-annual species common to all three lineages (Kal's Z-test, FDR-corrected $p<0.01$ and absolute fold change $>$ 1.5). Venn analysis revealed six miRNAs that were up-regulated in diapausing embryos in all three lineages (Fig 1D, Table S1): miR-10a/b/d, miR-101a, miR-146a and miR-192. Seven miRNAs were down-regulated in diapausing embryos in all three lineages (Fig 1E, Table 1): miR-130, miR.184, miR18a, miR-22a, miR-430a, miR-462, and miR-731. MiR-430 controls transition from maternal to zygotic transcription in fish eggs (Giraldez et al. 2006). This transition appears normal however in annual fish and well before diapause I, as assessed by onset of paternal transgene expression in N. furzeri (Fig. 2), suggesting a novel role for miR-430.

In C. elegans, some miRNAs are regulated in similar directions during diapause and aging. In particular miR-71, that is induced during diapause and is necessary for survival of diapausing larvae (Zhang et al. 2011). Expression of miR-71 increases with age and drops late in life (Pincus et al. 2011). Deletion of miR-71 results in shortened life span (de Lencastre et al. 2010) and the timing of miR-71 drop is predictive of individual worm lifespan (Pincus et al. 2011). In the annual species N. furzeri, we detected four miRNAs that are up-regulated during aging in brain liver and skin (Baumgart et al. 
bioRxiv preprint doi: https://doi.org/10.1101/017848; this version posted April 10, 2015 . The copyright holder for this preprint (which was not certified by peer review) is the author/funder, who has granted bioRxiv a license to display the preprint in perpetuity. It is made available under aCC-BY-NC-ND 4.0 International license.

2012). One of these miRNAs, miR-101a, is also up-regulated during diapause in all three annual lineages analyzed here. In human cancer cells, miR-101 represents a key node of a regulatory network with transcription factors and epigenetic modulators as the first neighbors and genes involved in cell-cycle progression as second neighbors (Huang et al. 2012). In addition, we detected conserved down-regulation of miR-18a during diapause. This miRNA is a member of the miR-17 92 genomic cluster, also known as oncomiR-1, that plays a major role in promoting cell proliferation in cancer cells (Olive et al. 2010). These miRNAs are down-regulated during aging of human mitoticallyactive cells (Grillari et al. 2010) and in brain and skin of $N$. furzeri (Baumgart et al. 2012). Regulation of miR-18a and miR-101a are therefore most likely related to mitotic arrest observed in diapausing killifish embryos (Meller et al. 2012). It is an interesting observation that the regulation of these miRNAs during diapause follows the same direction observed during aging and mirrors the regulation of miR-71 in C. elegans.

These results provide the first evidence that overlapping genetic networks control diapause and aging in vertebrates.

\section{Acknowledgments}

This work was partially supported by an internal grant of Scuola Normale Superiore and by the German Ministry for Education and Research (JenAge; BMBF, support code: 0315581A, 0315581C)

\section{Experimental Procedures}

Fish raising:

All the fishes used in this work were raised in $35 \mathrm{It}$ tanks $(25 \mathrm{~cm} \times 50 \mathrm{~cm} \times 28 \mathrm{~cm})$ filtered with an airdriven sponge filter at these densities:

- Aphyosemion australe, Aphyosemion striatum, Nothobranchius furzeri, Nothobranchius guentheri, Nothobranchius korthause, Scriptaphysemion guignardi and Epiplatys dageti: 3 or 4 couples each tank;

- Nothobranchius melanospilus: 1 or 2 couples each tank;

- Callopanchax occidentalis: 1 couple each tank;

- Rivulus cylindraceus, Rachovia brevis: 3 couples each tank

Water parameters used for all species

- $\mathrm{pH}: 7-8$;

- $\mathrm{KH}: 3-5$;

- $\mathrm{T}: 23-25^{\circ} \mathrm{C}$ 
$25 \%$ of the water in each tank was replaced once a week.

Fish feeding:

All the fishes were fed with granular food (Microgranuli, $\mathrm{SHG}^{\circ}$, Alessandria, Italy) twice a day and newly-hatched brine-shrimps (Premium Artemia, $\mathrm{SHG}^{\circ}$, Alessandria Italy) once a day (rising to three times a day will result in increased eggs production) for the amount they can eat in ten minutes.

Fish breeding:

Annual and non-annual fishes were bred using two different methods:

For annual fishes, a bowl (from $20 \mathrm{~cm} \times 15 \mathrm{~cm} \times 10 \mathrm{~cm}$ to $9 \mathrm{~cm} \times 9 \mathrm{~cm} \times 4 \mathrm{~cm}$ ), half-full of river sand $(\varnothing$ $0.3 \mathrm{~mm})$, was put on the bottom of the tank. More than one small bowl (0.7-1.3 for each male in the tank) worked better in tanks with more than 4 males. After a short period, or after a short conditioning (see below), fishes learned to go in the bowl and breed on the surface of the sand, laying and burying eggs deep into it.

Eggs could then be collected by removing the bowl from the tank, sieving the sand with a sieve $(1 \mathrm{~mm}$ grid) and then trasferring the eggs from the sieve to a Petri dish previously filled with tap water. Non-annual fishes were bred differently. $25 \mathrm{~cm}$ long "breeding mops" were made using $2 \mathrm{~mm}$ thick green/brown/grey wool (100\% acrylic), using a Bijou (by Sigma-Aldrich ${ }^{\circ}$ ) screwed into the top of each mop to make them floating. One or more mops (optimum is one each two males) were put in the tanks. After a short period, or after a short conditioning (see below), fishes learned to go in the mops and breed inside it, laying eggs on the wool filaments. Eggs could then be collected by removing the mops from the tanks and inspecting them, eggs were removed with one finger tips (eggs are adhesive and hard) and putting them into a Petri dish previously filled with tap water.

\section{Conditioning for breeding}

1) To force the breeding time is often useful a relative short period of habituation (from 5 days to 2 weeks), during which fish are put in a comfortable situation for coupling (with sand on the bottom of the tank or mops on the top), for a short period (1.5 to 3 hours) daily, at the same time of the day.

2) Intense breeding sessions (breeding fish every day for more than 5 days) would result in decreasing the amount of eggs produced. This can be solved by 1 or 2 days of rest and it is recommended to apply the period of rest before any significant experiments. Notice that if 
the non-breeding period is prolonged too much it can result in impaired eggs production when breedings are restored.

3) Fishes can be forced to produce eggs by separating males from females for several days (from 5 days to 2 weeks) and then putting them together in a tank with plenty of mops/sands.

4) The best setup for breeding is a 5-25 It (depending on species) tank with one male, two females and one mop/sand. This method works better in combination with point 3 while is often in conflict with point 1.

5) Rivulus cylindraceus breeding: Rivulus cylindraceus were able to produce eggs only after at least 10 days of gender separation, during which 4 males and 4 females are put into 2 different $40+$ It tanks set up with bricks where they can hide. Afterwards, they were put all together again in a 3 It tank with 5 mops.

RNA Isolation

For each species 20 eggs were pooled for each preparation. Total RNA was isolated using QIAzol (Qiagen) according to the manufacturer's protocol. $1 \mathrm{ml}$ cooled QIAzol and one $5 \mathrm{~mm}$ stainless steel bead (Qiagen) was added. Homogenization was performed using a TissueLyzer II (Qiagen) at $20 \mathrm{~Hz}$ for 2-3x $1 \mathrm{~min}$. After incubation for $5 \mathrm{~min}$ at room temperature $200 \mu \mathrm{l}$ chloroform was added. The tube was shaken vigorously for (at least) $15 \mathrm{~s}$ and incubated for $3 \mathrm{~min}$ at room temperature. Phase separation was achieved by centrifugation at $12,000 \times \mathrm{g}$ for $20 \mathrm{~min}$ at $4^{\circ} \mathrm{C}$. The aqueous phase was transferred into a fresh cup and $10 \mu \mathrm{g}$ of Glycogen (Invitrogen, Darmstadt, Germany), 0.16x volume $\mathrm{NaAc}(2 \mathrm{M} ; \mathrm{pH} 4.0)$ and $1.1 \mathrm{x}$ volume isopropanol were added, mixed thoroughly and incubated for 10 $\min$ at room temperature. The RNA was precipitated by a centrifugation step with $12,000 \times \mathrm{g}$ at $4^{\circ} \mathrm{C}$ for $20 \mathrm{~min}$. The supernatant was removed and the pellet was washed with $80 \%$ Ethanol twice and air dried for $10 \mathrm{~min}$. The RNA was resuspendet in $20 \mu \mathrm{l}$ DEPC-treated water by pipetting up and down, followed by incubation at $65^{\circ} \mathrm{C}$ for $5 \mathrm{~min}$. The RNA was quantified with a NanoDrop 1000 (PeqLab, Erlangen, Germany) and stored at $-80^{\circ} \mathrm{C}$ until use.

\section{Small RNA Sequencing}

For small RNA sequencing, small RNA cDNA libraries were prepared as follows: for each developmental stage equal quantities $(1 \mu \mathrm{g})$ of total RNA were submitted independently to Illuminas TruSeq small RNA sample preparation protocol (Illumina Inc., San Diego, USA). In brief, the library preparation was performed as follows: RNA was ligated with proprietary adapters to the $5^{\prime}$ and $3^{\prime}$ termini of the RNA. The adapter ligated samples were used as templates for cDNA synthesis. The cDNA was amplified with 13 PCR cycles to produce sequencing libraries, introducing specific 
nucleotide codes for indexing of libraries, to allow multiplexed sequencing. cDNAs were purified by 10\% Novex TBE polyacrylamide gel electrophoresis (Invitrogen) and eluted into $300 \mu$ l elution buffer (Illumina) for at least 2 hours at room temperature, to enrich for molecules containing inserts in the range of 18-33 nt. The resulting gel slurry was passed through a Spin-X filter (IST Engeneering Inc., Milpitas, CA, USA) and precipitated by the addition of $20 \mu \mathrm{g}$ glycogen, $30 \mu \mathrm{l}$ of $3 \mathrm{M} \mathrm{NaOAc}, \mathrm{pH} 5.2$, and $975 \mu \mathrm{l}$ of pre-chilled $\left(-20^{\circ} \mathrm{C}\right)$ ethanol. After washing with $70 \%$ ethanol, the pellet was dryed at $37^{\circ} \mathrm{C}$ for 5-10 min and dissolved in $10 \mu \mathrm{l}$ resuspension buffer (Illumina). The purified libraries were quantified on the Agilent DNA 1000 chip, diluted to $10 \mathrm{nM}$ and subjected to sequencing-by-synthesis on Illumina HiSeq 2000.

\section{Analysis of sequencing data}

Individual sequence reads with base quality scores were produced by Illumina sequencing. The data was analyzed by the use of CLC-worbench 4 (CLCbio, Arhus, Denmark). After eliminating reads with low quality and trimming the $3^{\prime}$ adaptor sequence, the remaining 18- to 33-nt reads represented by at least 30 sequence reads were grouped into unique sequence clusters. Annotation of sequence clusters was performed, allowing 2 mismatches and up to 2 additional bases at each end, by using 4 fish species as references from miRBase v16.0: Danio rerio, Tetraodon nigroviridis, Fugu rubripes, Oryzias latipes. Statistical significance was assessed using Kal'S Z test as implemented in CLCworkbech setting a p-adjusted value of 0.05 .

Hierarchical clustering and multi-dimensional scaling were performed using PAST (Hammer et al., 2001). Venn diagrams were generated using Venny (Oliveiros, 2007)

\section{References}

Baumgart M, Groth M, Priebe S, Appelt J, Guthke R, Platzer M , Cellerino A (2012). Age-dependent regulation of tumor-related microRNAs in the brain of the annual fish Nothobranchius furzeri. Mech Ageing Dev. 133, 226-233.

de Lencastre A, Pincus Z, Zhou K, Kato M, Lee SS, Slack FJ (2010). MicroRNAs both promote and antagonize longevity in C. elegans. Curr Biol. 20, 2159-2168.

Giraldez AJ, Mishima Y, Rihel J, Grocock RJ, Van Dongen S, Inoue K, Enright AJ, Schier AF (2006). Zebrafish MiR-430 promotes deadenylation and clearance of maternal mRNAs. Science. 312, 75-79.

Grillari J, Hackl M , Grillari-Voglauer R (2010). miR-17-92 cluster: ups and downs in cancer and aging. Biogerontology. 11, 501-506. 
Hrbek T, Larson A (1999). The evolution of diapause in the killifish family Rivulidae (Atherinomorpha, Cyprinodontiformes): A molecular phylogenetic and biogeographic perspective. Evolution. 53, 1200-1216.

Huang Y, Chen HC, Chiang CW, Yeh CT, Chen SJ, Chou CK (2012). Identification of a two-layer regulatory network of proliferation-related microRNAs in hepatoma cells. Nucleic Acids Res. 40, 10478-10493.

Kenyon C (2011). The first long-lived mutants: discovery of the insulin/IGF-1 pathway for ageing. Philos Trans R Soc Lond B Biol Sci. 366, 9-16.

Meller CL, Meller R, Simon RP, Culpepper KM , Podrabsky JE (2012). Cell cycle arrest associated with anoxia-induced quiescence, anoxic preconditioning, and embryonic diapause in embryos of the annual killifish Austrofundulus limnaeus. J Comp Physiol B. 182, 909-920.

Morin RD, O'Connor MD, Griffith M, Kuchenbauer F, Delaney A, Prabhu AL, Zhao Y, McDonald H, Zeng T, Hirst M, Eaves CJ , Marra MA (2008). Application of massively parallel sequencing to microRNA profiling and discovery in human embryonic stem cells. Genome Res. 18, 610-621.

Murphy WJ , Collier GE (1997). A molecular phylogeny for aplocheiloid fishes (Atherinomorpha, Cyprinodontiformes): the role of vicariance and the origins of annualism. Mol Biol Evol. 14, 790-799.

Olive V, Jiang I , He L (2010). mir-17-92, a cluster of miRNAs in the midst of the cancer network. Int J Biochem Cell Biol. 42, 1348-1354.

Pincus Z, Smith-Vikos T, Slack FJ (2011). MicroRNA predictors of longevity in Caenorhabditis elegans. PLoS Genet. 7, e1002306.

Wourms JP (1972). Developmental biology of annual fishes. I. Stages in the normal development of Austrofundulus myersi Dahl. J Exp Zool. 182, 143-167.

Zhang X, Zabinsky R, Teng Y, Cui M , Han M (2011). microRNAs play critical roles in the survival and recovery of Caenorhabditis elegans from starvation-induced L1 diapause. Proc Natl Acad Sci U S A. 108, 17997-18002. 


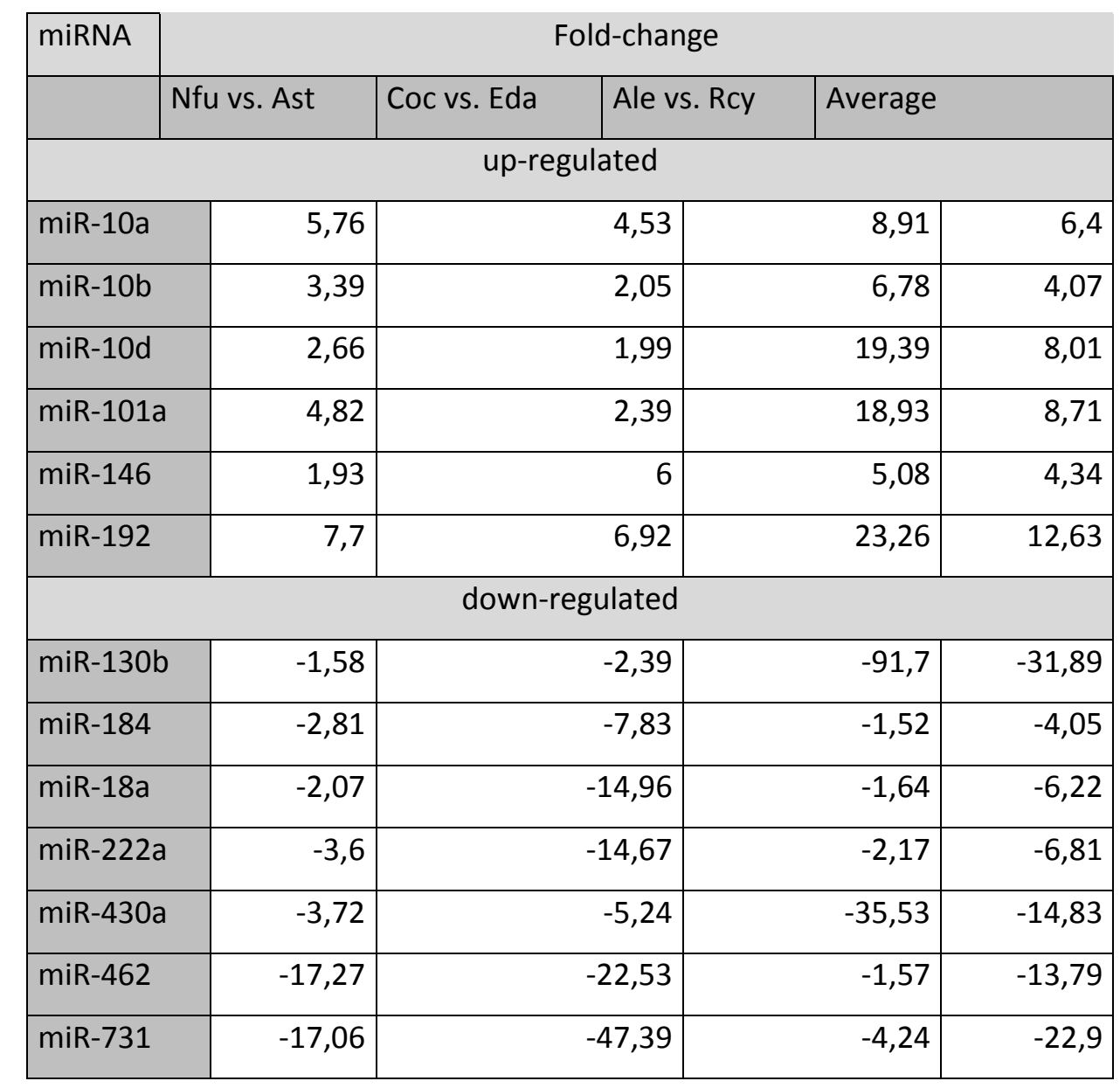

Table 1. Fold-changes of miRNAs that were detected as differentially-expressed in all three lineages. The miRNA name indicates the $D$. rerio miRNA that was used for annotation. Species codes: $\mathrm{Nfu}=$ Nothobranchius furzeri, Ast $=$ Aphyosemion striatum, Coc = Callopanchax occidentalis, Eda = Epiplatys dageti, Ale = Austrofundulus leohoignei, Rcy = Rivulus cylindraceus.

\section{Figure Legends}

Figure 1 (A) Cladogram of killifish depicting the species used in this study. Red branches represent annual genera and blue branches non-annual genera. (B) MDS plot of all analyzed samples. The red dots represent annual genera and blue dots non-annual genera.

Nothobranchius-1 and -2 are biological replicates. (C) Hierarchical clustering of the same samples as in (B), Bray-Curtis dissimilarity index. (D,E) Venn plot of common up-and down- 
bioRxiv preprint doi: https://doi.org/10.1101/017848; this version posted April 10, 2015 . The copyright holder for this preprint (which was

not certified by peer review) is the author/funder, who has granted bioRxiv a license to display the preprint in perpetuity. It is made available under aCC-BY-NC-ND 4.0 International license.

regulated miRNAs, respectively. Each contrast was performed between annual- and nonannual species of the same evolutionary lineage. Species codes: Nfu = Nothobranchius furzeri, Ast $=$ Aphyosemion striatum, $\mathrm{Coc}=$ Callopanchax occidentalis, $\mathrm{Eda}=$ Epiplatys dageti, $\mathrm{Ale}=$ Austrofundulus leohoignei, Rcy = Rivulus cylindraceus.

Figure 2 Expression of paternal transgene in N. furzeri. The expression of paternal genes is shown in a transgenic line expressing eGFP under the ubiquitin promoter. The top two rows illustrate a transgenic egg and the bottom two row illustrate a control from the same clutch. The dotted lines indicate the boundary of the embryo. Dispersed cells are shown in the dispersed state ( $70 \%$ epiboly). Please note that the first expression of the transgene is observed at the dome stage when mid-blastula transition is expected. 
A

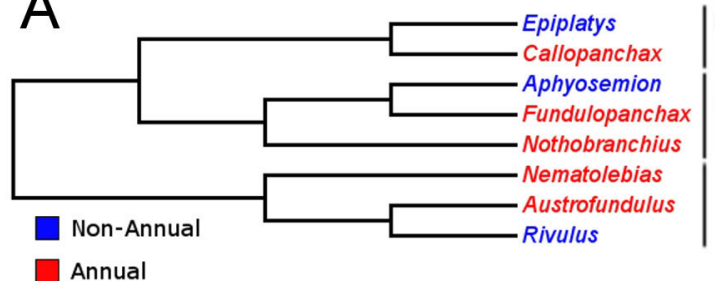

Annual

0

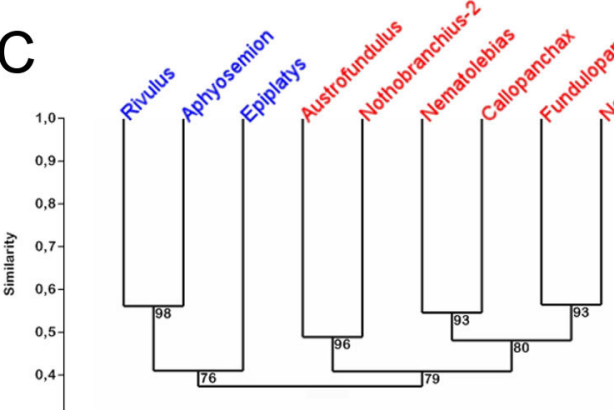

D

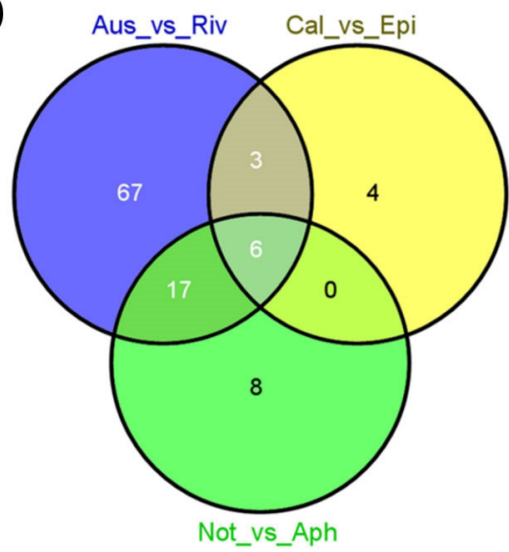

B

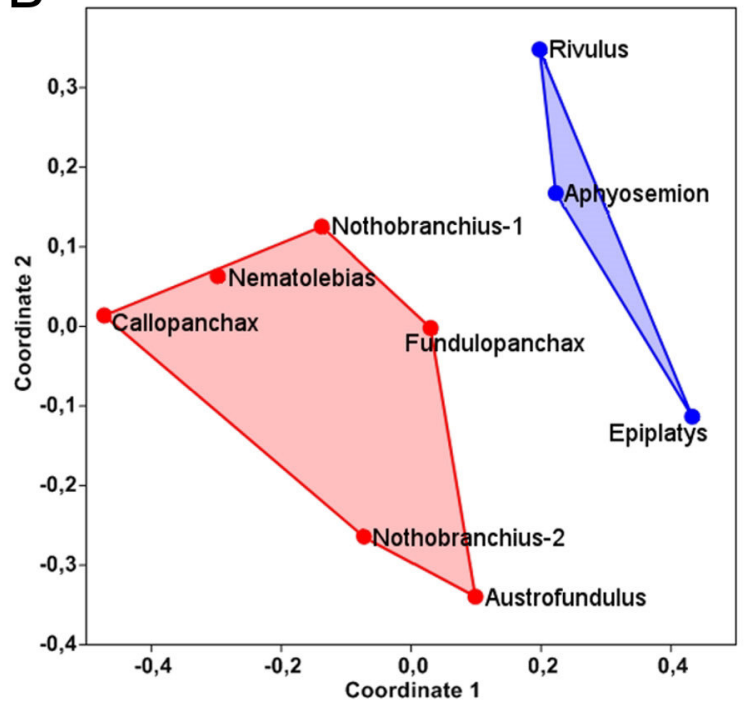

E

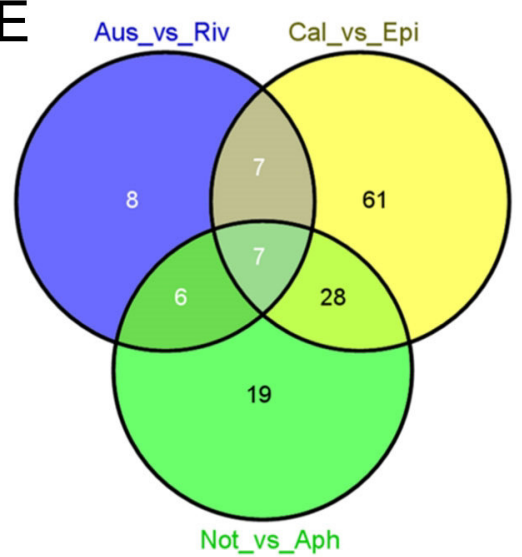

mir-130b mir-184 mir-18a mir-222 mir-430a mir-462 mir-731 
2 Cells

1 Day P.F.

70\% Epiboly Diapause I Diapause II

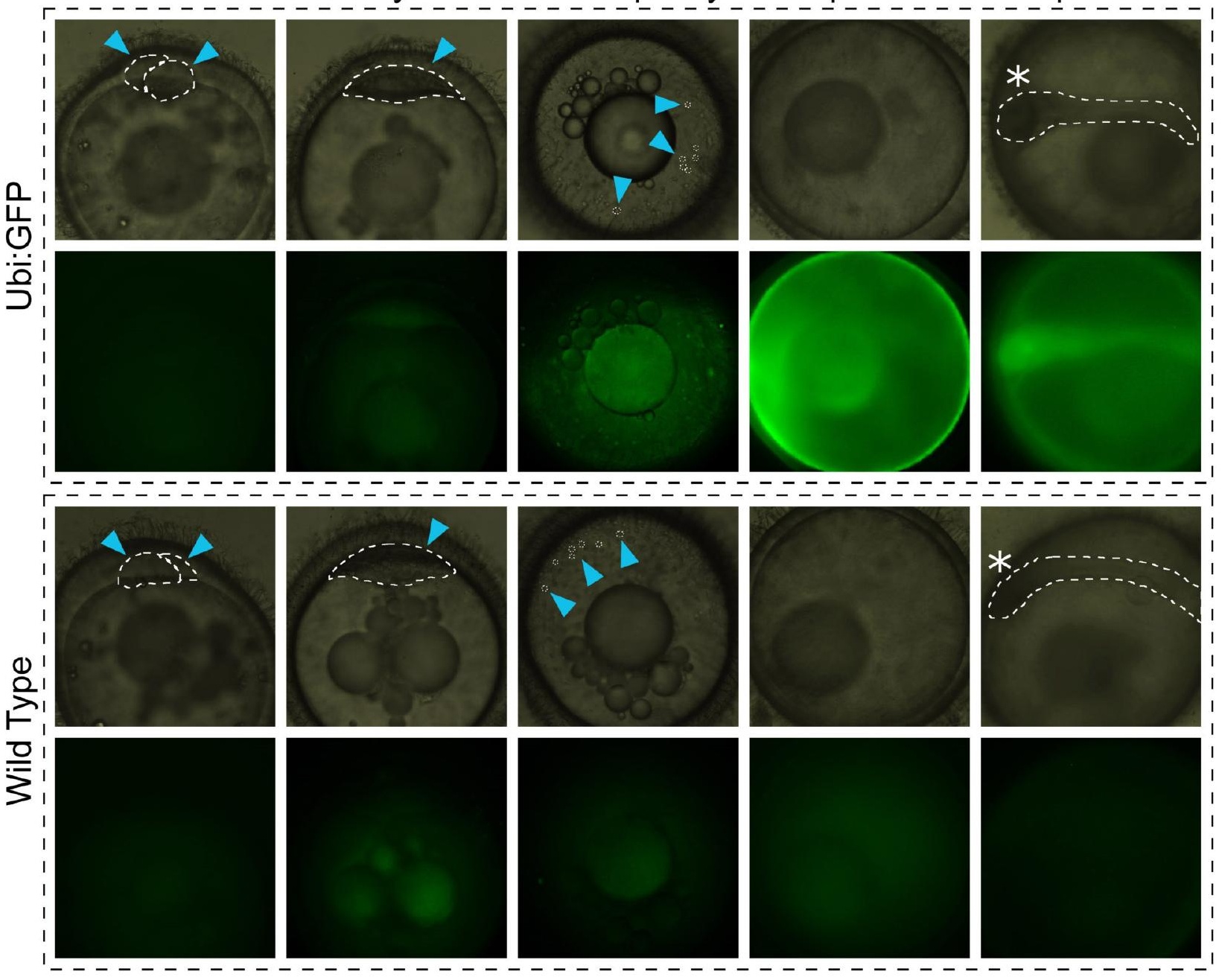

